\title{
Dessine-moi un cheminot. Images, imaginaire et représentations des cheminots belges
}

'Please, draw a railwayman for me'. Images, symbols and representation of

Belgian railway persons

Paul J.G.M.J. Van Heesvelde

\section{OpenEdition}

\section{Journals}

Édition électronique

URL : https://journals.openedition.org/rhcf/172

DOI : 10.4000/rhcf.172

Éditeur

Rails \& histoire

Édition imprimée

Date de publication : 1 décembre 2007

Pagination : 351-367

ISSN : 0996-9403

\section{Référence électronique}

Paul J.G.M.J. Van Heesvelde, « Dessine-moi un cheminot. Images, imaginaire et représentations des cheminots belges », Revue d'histoire des chemins de fer [En ligne], 36-37 | 2007, mis en ligne le 10 mai 2011, consulté le 22 avril 2022. URL : http://journals.openedition.org/rhcf/172 ; DOI : https://doi.org/ $10.4000 /$ rhcf. 172

Ce document a été généré automatiquement le 22 avril 2022

Tous droits réservés 


\section{Dessine-moi un cheminot. Images, imaginaire et représentations des cheminots belges}

'Please, draw a railwayman for me'. Images, symbols and representation of Belgian railway persons

Paul J.G.M.J. Van Heesvelde

\section{NOTE DE L'ÉDITEUR}

La diffusion de certaines illustrations de cet article n'est pas autorisée sur l'Internet.

Les lecteurs sont invités à consulter l'édition imprimée de la Revue d'histoire des

chemins de fer

pour prendre connaissance de l'article complet

1 L'analyse des images, photos ou films présentant des cheminots n'a pas encore été faite en Belgique. Dans le cadre de notre série de colloques et journées d'étude, il est donc important de présenter à titre de préliminaire une vue cohérente de l'image et l'identité cheminote belge. Cet exposé ne donne qu'un premier aperçu d'un champ de recherche resté inexploité. Cela dit, des indices convergents nous permettent d'affirmer que l'image belge du cheminot n'est pas très éloignée de l'image française de la même corporation. Si les apparences sont trompeuses en France, comme Michel Ionascu l'indique à propos du cinéma, elles le sont aussi en Belgique puisque toute représentation est d'abord une construction : «Qu'il soit beau ou laid, le vrai, s'il existe, se présente donc sous la forme d'un faux ${ }^{1}$. »

2 Un autre élément à prendre en compte est le constat auquel je parviens en rédigeant cet article : il s'agit d'un échange de métaphores dans la façon d'écrire l'histoire. Dans le titre déjà, je "dessine” un cheminot, bien que j'ai paraphrasé l'expression "dessinemoi un mouton" employée par Saint-Exupéry dans Le Petit Prince. La relation complexe 
qu'entretiennent les arts visuels, les arts plastiques et l'historiographie n'est qu'une explication partielle de ce phénomène. Il s'agit de traduire à l'aide du langage écrit, en recourant le plus souvent à la métaphore, les métaphores que l'artiste, l'artisan ou le photographe ou dessinateur amateurs ont eux-mêmes mises en œuvre, en un texte qui a la possibilité d'exprimer l'imaginaire de l'image et qui d'en faire une interprétation. Cette construction de la représentation dépend du cadre de référence propre à l'auteur dans lequel elle s'inscrit ; chaque représentation est donc une interprétation. Dans mon cas personnel, l'image n'est pas neutre et sera influencée par le fait que j'ai travaillé à la SNCB (Société nationale des chemins de fer belges) comme cheminot parmi les cheminots. Cependant ce niveau d'analyse ne doit pas être considéré comme une contrainte supplémentaire.

3 Il est intéressant en effet de voir comment les chemins de fer et leur personnel sont (re)présentés, et quelle évolution nous pouvons constater dans ces images. L'exercice est moins évident qu'il ne semble l'être; les chemins de fer n'ont pas une tradition de représentation du personnel dans l'iconographie. Dans sa recherche concernant le film français, Michel Ionascu constate que « le monde ferroviaire est souvent obsédé par le bon fonctionnement technique de ses machines et engins en tout genre: locomotives, locotracteurs, postes d'aiguillages [...]. De l'extérieur, cet univers est assimilé à une superstructure vivant 24 heures sur 24 , d'une manière quasi automatique, nuit et jour, et par tous les temps (ou presque). Mais le voyageur, voit-il vraiment les cheminots? $»^{2}$. Si l'on veut parvenir à la reconstruction d'une identité professionnelle à l'aide de l'image, il faut inclure le résultat d'autres recherches, par exemple une histoire orale. Le monde scientifique en Belgique, qu'il s'agisse de sociologues ou d'historiens, n'a pas entrepris de recherches dans le milieu cheminot. Cette lacune, combinée au manque d'images comme de leur analyse, vaut aussi pour la littérature et l'image créées par les écrivains.

4 Si une analyse approfondie des convergences et divergences des métaphores présentes dans l'image et le langage reste à faire, la lecture d'une anthologie de poésie et littérature des chemins de fer donne pour premier résultat le fait que le cheminot est invisible et le travail à peine représenté, à part quelques rares exemples ${ }^{3}$. Le sujet de prédilection de la littérature cheminote $\mathrm{du} \mathrm{XIX}^{\mathrm{e}}$ et du début du $\mathrm{Xx}^{\mathrm{e}}$ siècles est le paysage et l'interaction avec le voyageur. Un autre thème est bien évidemment la technique, mais il est impossible de faire un dessin, même une esquisse en s'appuyant sur la description littéraire d'un engin. La machine a des roues, les auteurs le disent clairement, mais combien en a-t-elle ? Est-ce une machine à quatre roues? Ou à sept ? Impossible de le savoir, car la poésie veut que l'on traite le sujet en utilisant des métaphores ; on ne maitrise pas la technologie, seule le rapport de cause à effet entre le coup de fouet donné au cheval et la vitesse obtenue est connu. On ne retrouve pas le même rapport quand le mécanicien ouvre le régulateur pour faire démarrer sa machine.

5 Nous nous limitons donc ici à l'analyse de quelques photographies, qui toutes escamotent les cheminots plus qu'elles ne les dévoilent. J'attirerai ensuite l'attention sur la façon dont l'image de quelques professions cheminotes est entrée ces dernières années dans les publications officielles de la SNCB. Je vérifierai ensuite ce qui reste de la mémoire collective, de la création d'identité, de l'esprit d'équipe et de la culture d'entreprise antérieurs dans une société de transports qui s'est scindée, sous la pression de la législation européenne, en trois entreprises distinctes, une société 
holding (SNCB-Holding), un gestionnaire d'infrastructures (Infrabel) et un opérateur de transport (SNCB). Depuis quelques années en effet des photographies de certaines catégories de cheminots apparaissent dans rapport annuel de la société. Dans ce cas également, on peut se demander si la façon de représenter les cheminots transmet une image exacte des hommes et des femmes qui font circuler les trains. À défaut d'un corpus riche de grandes séries, il faut utiliser d'autres types de sources pour construire une image dont la cohérence sera finalement plus ou moins assurée.

\section{"Ceci n'est pas un cheminot ». Entre image et représentation des cheminots belges}

6 Si l'abondance des sources picturales pour l'historiographie des chemins de fer semble un atout, il est clair que l'image de l'homme ne participe pas à cette richesse. Non seulement le cheminot est invisible, mais le cheminot au travail semble entièrement inexistant. "L'outil technologique dissimule un être humain qui ne serait, finalement, qu'un élément technique ou organisationnel entièrement dévoué à la survie d'une pieuvre industrielle ${ }^{4}$.» La vaste collection de photographies de gares de Hugo De Bot récemment publiée confirme cette impression que nous donne Michel Ionascu. Chacun sait qu'au chemin de fer, on n'a presque pas de personnel! La plupart des photos montre ou la façade de la gare vers la ville ou la façade sur les voies, avec les platesformes et les quais, mais ces quais sont déserts et le personnel, invisible, doit être réfugié dans les bâtiments ou les bureaux. Les images où le personnel est manifestement présent sont souvent intitulées : «La gare et son personnel. » On le voit dispersé sur les quais, réuni autour du chef de gare ou d'un engin. Il est rare que le personnel prenne place devant la façade extérieure, sur le parvis de la gare, car dans ce cas les cheminots ont quitté leur "habitat " normal, la gare considérée comme un élément interne au système ferroviaire. La gare constitue le passage entre ces deux mondes. Les publications de Hugo De Bot sont très précieuses pour leurs informations sur l'histoire de l'architecture des gares belges et constituent un véritable inventaire des bâtiments, mais il faudrait revenir aux originaux pour risquer une analyse approfondie ${ }^{5}$. Étant donné le format de publication il n'est par exemple pas toujours facile d'identifier les figures que l'on aperçoit sur les quais comme appartenant au personnel, ou comme des voyageurs ou de simples passants. À première vue le personnel des gares est présent dans moins de $10 \%$ des photographies. Dans certains cas, pourtant, la qualité de l'image est plus grande, le détail plus visible et surtout, l'information plus évidente : un aiguilleur se repose sur les leviers pour manœuvrer les aiguillages ou la manœuvre d'un lorry dans une gare. Mais ce type d'image est extrêmement rare, puisque il montre des gens au travail. Pour revenir au cas général, il est clair que les chemins de fer n'ont pas besoin de visualiser leur personnel; autrement dit, le bon cheminot est le cheminot invisible. Mais s'il en est ainsi, qui s'occupe de maintenir l'ordre, d'appliquer les règles et de maintenir la discipline? Une affiche de février 1939 nous renseigne en ce qui concerne la sécurité des passages à niveau : un boy-scout montre le feu rouge interdisant tout passage et attire l'attention sur le phénomène : « un train peut en cacher un autre. » Cet élément d'une campagne de sensibilisation et de sécurité routière confirme les résultats obtenus par Michel Ionascu à propos du cinéma pour ce qui est de l'invisibilité du cheminot. La question clef est autre : le cheminot est-il invisible à cause du caractère technique du sujet et de 
la volonté de marquer (par défaut) ce caractère, ou l'est-il pour d'autres raisons ? Le cheminot aurait-il une image négative? Les sources écrites ne sont pas décisives à ce propos, mais il ressort du roman Het Rollende Leven (La Vie roulante - ma traduction), de Gustaaf Vermeersch, que le cheminot n'est pas toujours bien vu. Dans les années 1970 le rock band hollandais Shocking Blue enregistre la chanson : « Never marry a railroad man, he loves you every now and then, his heart is at his new train."

7 Figure 1. «Opgelet! » (Attention!), affiche éditée par la SNCB, février 1939.

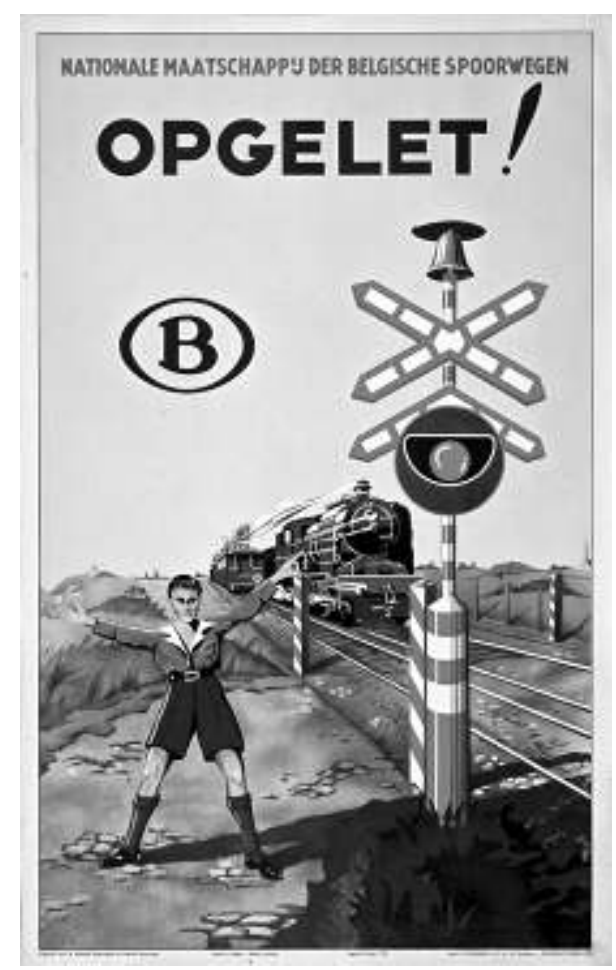

SNCB Holding, Archives et photothèque. CSNCB Holding

8 Autrement dit, il est impossible de déterminer avec certitude dans quelle mesure la représentation du cheminot a contribué à construire une identité cheminote à l'intérieur du groupe social lui-même. Pour ma part, je suis plutôt persuadé du fait que les photographies, les affiches et l'iconographie en général ont contribué davantage que la mémoire collective, le partage de valeurs communes ou des mêmes expériences à la construction du groupe social. Je précise mon point de vue grâce à deux autres images. La première montre Gustaaf Vermeersch, accompagnateur de trains et « chefgarde » (chef de train) aux chemins de fer de l'État ${ }^{6}$. 
Figure 2. L'écrivain cheminot Gustaaf Vermeersch

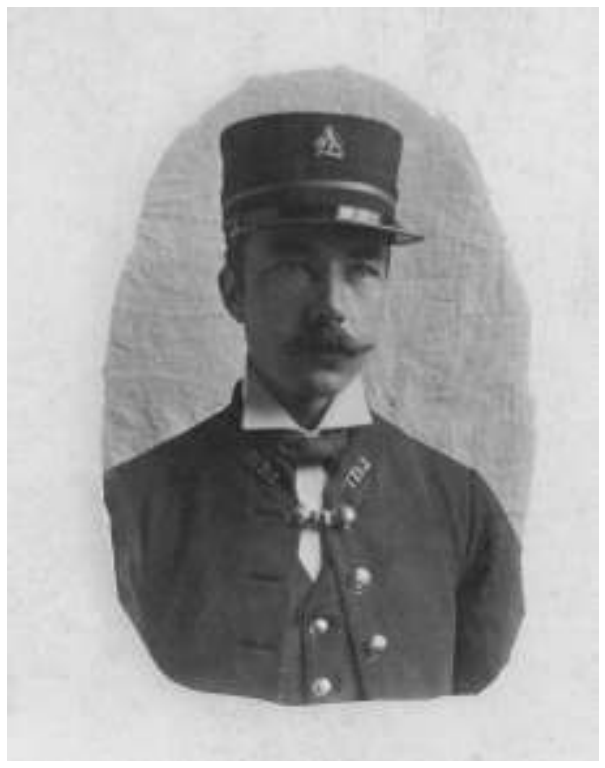

Photographie. AMVC (Archief en Museum voor het Vlaamse Cultuurleven), Letterenhuis, Anvers. () AMVC, Letterenhuis, Anvers.

9 Nous ne disposons que de quelques photos de Gustaaf Vermeersch, la plupart en civil. La question est: "qui est représenté? » S'agit-il du cheminot Vermeersch, du syndicaliste Vermeersch, de l'écrivain Vermeersch ou du flamingant Vermeersch? Quelle est l'image qu'a souhaité donner Vermeersch de lui-même? J'ai tendance à penser qu'il se sentait plutôt écrivain, syndicaliste et se considérait comme le défenseur du personnel flamand de la fonction publique. Il est difficile de le prouver, car il faudrait d'abord pouvoir répondre à la question suivante: pourquoi était-il syndicaliste? Par amour de son métier, par sentiment d'appartenance à un groupe ou pour intérêt propre? Son roman autobiographique La Vie roulante donne des indications sur les difficultés des relations sociales entre les différents métiers cheminots et la formation d'une identité propre de chef-garde. Le chef-garde Vermeersch a, comme ses collègues et comme je l'ai porté pendant une certaine période, un deuxième prénom : chef. La question reste donc posée : qui est représenté sur cette photo ? C'est le cadre de référence du spectateur qui définit le personnage. Les tenants du monde littéraire verront probablement l'auteur, tandis que les syndicalistes reconnaîtront le fondateur d'un syndicat flamand et les défenseurs de la cause flamande y verront Vermeersch le flamingant. Peu de gens verront en lui le cheminot.

La même considération vaut pour la photographie suivante, qui met en évidence des cheminots de différentes professions dans un cortège politique où ils défilent pour le parti socialiste en 1924. À ce moment, le parti socialiste est très présent et le syndicat socialiste s'unifie; les différents syndicats professionnels se réunissent autour de la commission syndicale, qui prend plus tard le nom de syndicat socialiste. Les hommes se retrouvent autour d'un engin, représenté au milieu de la photographie. Cette photographie est emblématique de l'ordre naturel des choses qui à ce moment soumet le cheminot à la technique. Cependant, il est plus important de remarquer que dans cette image les manifestants - cheminots - n'ont pas dans leurs rangs de conducteurs, du moins si mon analyse des uniformes est juste. La machine, symbole de leur profession et de leur existence, n'est pas accompagnée par les hommes qui la 
conduisent, mais bien par le personnel de surveillance et par les ouvriers. La description de relations tendues entre chefs de train et personnel de surveillance par Gustaaf Vermeersch, est en partie la réalité des années vingt et est visualisé dans cette photo.

Figure 3. $40^{\mathrm{e}}$ anniversaire du Parti ouvrier belge (POB) à Bruxelles en 1924, défilé du syndicat national des cheminots.

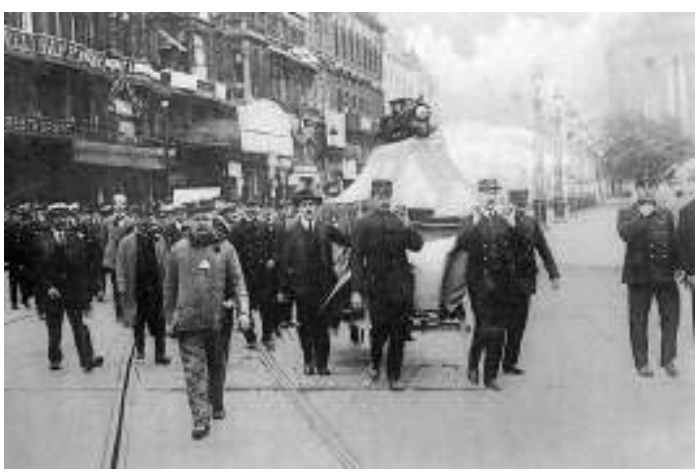

Archief en Museum van de Socialistische Arbeidersbeweging (AMSAB) - Instituut voor Sociale Geschiedenis (ISG), Gand.

\section{Quelle image pour quel cheminot?}

11 Cette image a évolué pendant soixante-dix ans ; au début des années 1990, période dans laquelle je suis devenu cheminot, j'ai découvert un tout autre chemin de fer. La SNCB de 1924 ne se compare plus à la société de 1992 et le chemin de fer de 2003 est tout autre que celui que j'ai vécu. Mais il n'y a pas de rupture sans constante. J'ai commencé ma carrière à la gare de Schaerbeek, qui était à l'époque la gare la plus importante du réseau; elle offrait tous les services: voyageurs, marchandises, messagerie, trains internationaux, triage, ateliers de traction (électrique et diesel), ateliers de réparation de wagons et de voitures, différents services d'entretien (voie, signalisation, caténaires), laboratoire pour le contrôle de la qualité des achats, etc. La gare était dans les années 1930 une des plus modernes, mais au début les années 1980 elle respirait l'histoire. À certains endroits, le vécu pesait sur les épaules et la mémoire collective y était omniprésente. Les années 1990 ont été aussi une période de restructuration, qui n'était pas à l'initiative de la part de la société elle-même: l'Europe menait une politique contraignante à l'égard des entreprises publiques nationales. Le personnel gagne en visibilité, tout comme le voyageur. La culture d'entreprise a forcément changé et ce qui reste de l'identité cheminote antérieure n'est pas clair. D'après mon vécu, cette identité professionnelle n'avait guère changé en 1992. Il est bien possible que cette période de restructurations ait ranimé la mémoire collective. À l'autre bout de la chaîne, en 2005, on trouve les images d'une société de transport qui fonctionne dans une logique économique différente et où le voyageur, le client occupent une position centrale, où le cheminot agent commercial des trains sera présent et visible. La question de l'identité se pose bien entendu : que reste-t-il de ces différents cheminots dans nos mémoires ? Comment a évolué la culture d'entreprise ? C'est difficile à dire, puisque nous ne disposons pas de points de repères ; aucune étude n'a été effectuée à ce sujet. Je peux m'appuyer sur mon propre vécu et sur mes notes journalières, qui 
consignent des particularités de mon passage aux chemins de fer. Ces annotations marquent la lente modification de la culture d'entreprise.

Je suis entré à la SNCB en 1992, et ma première vision en fut un sentiment de " déjà $v u »:$ j'ai retrouvé en partie le chemin de fer de mon enfance dans une gare déserte, à l'exception du guichetier et du porteur. La salle des pas perdus était vide; quelques passants de temps à autre. Les collègues étaient aussi invisibles qu'ils l'étaient quand j'étais enfant et pour la première fois je comprenais d'où venait cette invisibilité : il y avait beaucoup de travail à faire - ailleurs - pour que les trains circulent. Cela n'a pas empêché que mes premiers contacts avec les cheminots ne fussent peu amènes : mes nouveaux collègues - cheminots doués - me regardaient d'un mauvais œil, puisque je ne connaissais pas le service des trains et surtout que je mangeais mes tartines quand j'en ressentais le besoin. Au chemin de fer, on connaît au moins les heures de passage et la destination des trains et on ne mange pas parce qu'on a faim, mais parce que «c'est l'heure » : il est midi.

Une réaction à la réponse que j'ai donnée à une question - classique - que l'on m'a posée à mon arrivée ne cesse de m'intriguer. Mes collègues voulaient toujours savoir où les nouveaux embauchés avaient travaillé auparavant et, surtout, s'ils venaient ou non «du secteur privé ». Dans mon cas, la réponse était négative, puisque j'avais travaillé dans la fonction publique, aux Archives générales du Royaume. "Ah, le privé, donc!", me répondaient-ils en ouvrant de grands yeux. J'insistais sur le fait qu'il s'agissait de la fonction publique. Alors venait une réponse décisive : «La fonction publique, c'est la $\mathrm{SNCB}$, le reste, c'est le privé.» Je me retrouvais donc dans le dernier carré des défenseurs de la patrie, de l'État unitaire et du service public. D'où venait cette réaction hostile à l'égard des "gens du privé "? Les années 1980 et 1990 sont marquées par la lente désintégration de la Belgique unitaire, en application d'une suite de réformes d'État. Les sociétés de transporteurs en commun urbain et péri-urbain sont régionalisées en 1990 (région flamande, région wallonne, région de Bruxelles), mais la SNCB reste une institution fédérale. En 1991, la SNCB est de plus transformée en société anonyme de droit public et la Commission européenne publie la directive 91/440, point de départ d'une libéralisation des réseaux de chemins de fer. "Libéralisation », dans le milieu cheminot, signifie menace de privatisation. Le monde extérieur menace le statut des cheminots. L'évolution de l'effectif du personnel montre que sa baisse est réelle entre 1990 et 1998. 


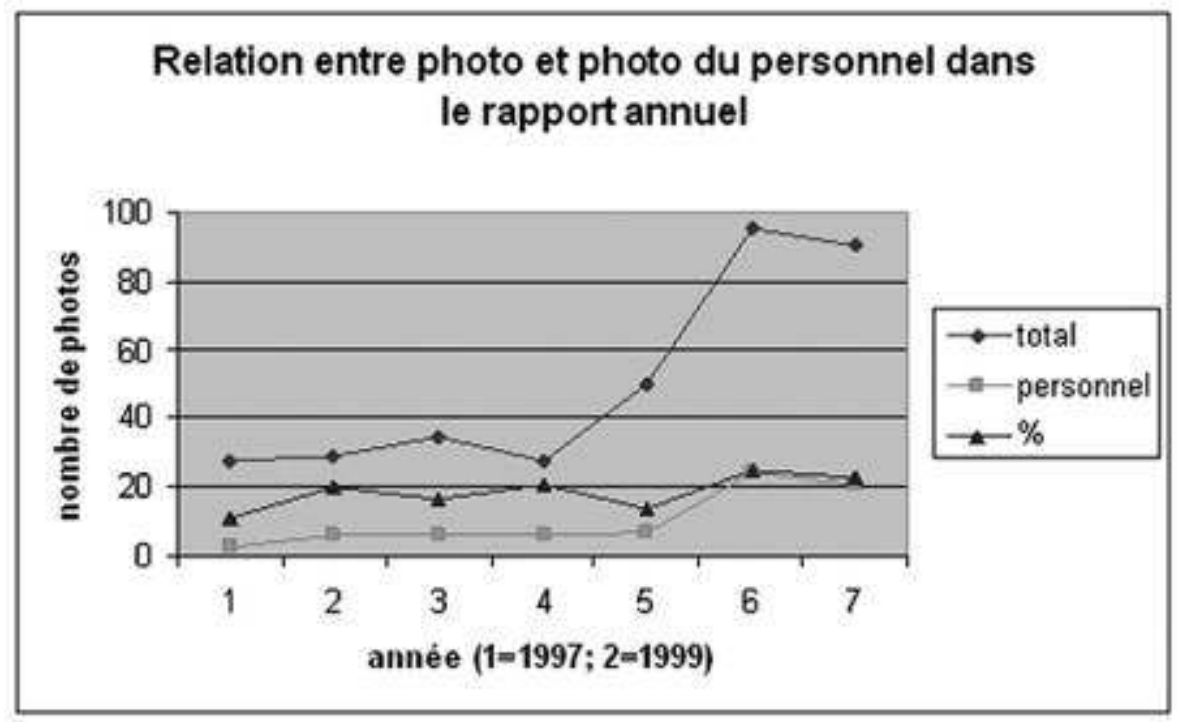

Source : Statistiques SNCB, édition 2003.

Je me retrouvais donc dans un groupe qui était prêt à défendre son statut, son travail, sa situation professionnelle et sa culture d'entreprise. Les cheminots m'apprennent le métier, ses secrets, le groupe m'apprend la solidarité et bien entendu ses tabous. Après quelques semaines, je suis toujours cheminot, mais j'ai découvert l'existence prédominante de groupes différents, de professions et métiers cheminots différents qui constituent la communauté - voire la corporation des cheminots, dans tous leurs grades et leurs qualités. Un sous-chef ne s'entend pas forcément bien avec un aiguilleur ni celui-ci avec un enrayeur, et vice versa. Je reconnais la justesse des analyses de Vermeersch vieilles d'un siècle. Dès les premiers jours, je suis confronté à un mythe : l'agent de triage écrasé entre deux wagons. C'est le mythe urbain par excellence, qui met en évidence l'héroïsme et la solidarité dans le moment le plus pénible dans la vie, celui de la mort atroce. Le mythe veut que l'enrayeur est encore vivant entre les tampons, et ses collègues lui donnent une dernière cigarette, avant de le libérer, parce qu'on sait qu'il va mourir dès que les deux wagons seront séparés. Après cette cigarette et après, selon certaines sources un dernier mot à son épouse, il meurt au moment où il est libéré de la machine, quasi dans les bras de ses collègues. Ce mythe urbain m'a été raconté à maintes reprises non seulement par des cheminots, mais aussi par d'autres, non cheminots. Une constante de cette histoire est que personne ne peut dire où cet accident s'est produit, ni en quelle année. Ma recherche dans les dossiers d'accidents est restée sans résultat. Une autre constante est que certains cheminots étaient vexés quand je demandais comment il était possible qu'une personne aux poumons écrasés puisse fumer. C'était une offense, parce qu'un nouveau venu dans le groupe touchait à l'héroïsme. Ce mythe met en évidence la solidarité ; au chemin de fer on ne meurt pas seul et on prend soin de la veuve et orphelins. D'autres mythes urbains de Schaerbeek tiennent plus à la virilité du cheminot, des histoires qui ne se racontent qu'entre hommes et entre cheminots. Mais il ne s'agit pas uniquement de mythes; la construction d'un passé commun aide à intégrer les nouveaux.

Après mes neuf mois de formation au service du mouvement, je prends donc mon service à Schaerbeek, alors comme je l'ai dit une des plus grandes gares de la Belgique. 
Bientôt, je monte en cabine d'aiguillage, muni de ma connaissance approfondie de la réglementation et du jargon cheminot mais je constate très vite qu'on ne nous a pas appris le jargon des aiguilleurs. Un nouveau sous-chef ne peut pas comprendre leurs échanges, tant qu'il n'est pas "initié ». J'entends pour la première fois parler des différents noms utilisés pour les voies ou les directions : le "kapper", la voie 12 dans les différents faisceaux de la gare de Schaerbeek, nom dont l'origine est inconnue, ou le "Warmenhoek", dont la connotation sexuelle est claire; ce soi-disant "quartier chaud»- une destination de train -, existait dans d'autres gares, notamment à Merelbeke, ou les aiguilleurs l'appelaient les «Warme Landen ». D'autres destinations tiennent leur nom de la fonction ou d'un équipement antérieur: le mélangeur, qui date de la période de la vapeur, a disparu depuis déjà plus de vingt ans, mais le nom est maintenu pour indiquer l'arrêt de la rame de service. Un cas particulier est l'itinéraire "Ingénieur ", l'itinéraire le plus simple à tracer, pour lequel on n'a besoin que d'une seule manette, pour en même temps l'enclencher et ouvrir le signal. Un ingénieur est donc capable de faire partir ce train ce qui sous-entend que les autres itinéraires demandent la compétence d'un aiguilleur. Le nom « Ingénieur » renvoie au mépris des agents pour la direction, jadis dominée par les ingénieurs, mépris qui n'existe plus. Probablement les agents ne savent-ils plus interpréter l'origine de cette dénomination, comme on ne peut pas expliquer l'insulte entre aiguilleurs : "Va travailler au tram !", qui indique clairement à l'insulté qu'il n'est pas un cheminot mais un «petit cheminot».

\section{Et la réalité ?}

Si le personnel aide à construire une culture d'entreprise et une identité, l'entreprise y contribue également. Depuis quelques années le voyageur est plus visible et avec lui le « chef-garde » (chef de train), entre temps devenu « accompagnateur de train » (agent commercial ou contrôleur).

Il est bien évident que ce qui précède est le résultat des mentalités des différents groupes présents dans la SNCB. Il est même vraisemblable que les résultats seront différents si d'autres groupes sont interrogés. Mais quelques éléments communs ressortent néanmoins, dont nous pouvons tenter une synthèse. Nous sommes devant un groupe de personnes qui se donnent la qualité de cheminot; on est d'abord cheminot parce qu'on est reconnu comme cheminot par ses collègues. Pour ma part je me sens encore cheminot, mais je ne suis plus considéré comme un cheminot à cent pour cent. Dans cette population cheminote, il y a probablement plus de 100 métiers et professions différents couverts par la notion de cheminot : médecin, architecte, cadre, agent commercial train, conducteur, aiguilleur, enrayeur, etc. La formation des cheminots comprend aussi bien l'université que l'école des porteurs jusqu'aux années 1980. Le tableau 1 donne la répartition des effectifs entre les différentes unités de l'entreprise en 2003.

Tableau 1. La répartition du personnel de la SNCB entre unités de l'entreprise en 2003.

\begin{tabular}{|l|l|}
\hline Staff et audit interne & 1390 \\
\hline Finances & 386 \\
\hline
\end{tabular}




\begin{tabular}{|l|l|}
\hline Ressources humaines & 669 \\
\hline Opérations & 277 \\
\hline Fret & 449 \\
\hline Voyageurs & 4876 \\
\hline Matériel & 6840 \\
\hline Train & 5803 \\
\hline Réseau & 9404 \\
\hline Infrastructure & 8430 \\
\hline Patrimoine & 1854 \\
\hline Service médical & 163 \\
\hline Personnel détaché & 520 \\
\hline
\end{tabular}

Source : Statistiques SNCB.

18 La répartition par tranche d'âge est très homogène, puisqu'environ 50 \% de l'effectif est âgé en 2003 de 40 à 55 ans. L'évolution de la répartition par sexe montre une baisse relative du nombre d'hommes dans la dernière décennie. Dans les faits, la SNCB est une société masculine, mais la présence des femmes s'affirme dans l'image de l'entreprise.

Tableau 2. Répartition du personnel de la SNCB par sexe, évolution 1997-2004 (nombre d'agents).

\begin{tabular}{|l|l|l|}
\hline Année & Personnel masculin & Personnel féminin \\
\hline 1997 & 39721 & 1868 \\
\hline 1998 & & \\
\hline 1999 & 38992 & 2545 \\
\hline 2000 & 38831 & 2832 \\
\hline 2001 & 39587 & 3142 \\
\hline 2002 & 69614 & 3294 \\
\hline 2003 & 38010 & 3051 \\
\hline 2004 & 32262 & 3027 \\
\hline
\end{tabular}

Source : SNCB, Rapports annuels, 1997-2004. 
(nombre d'agents).

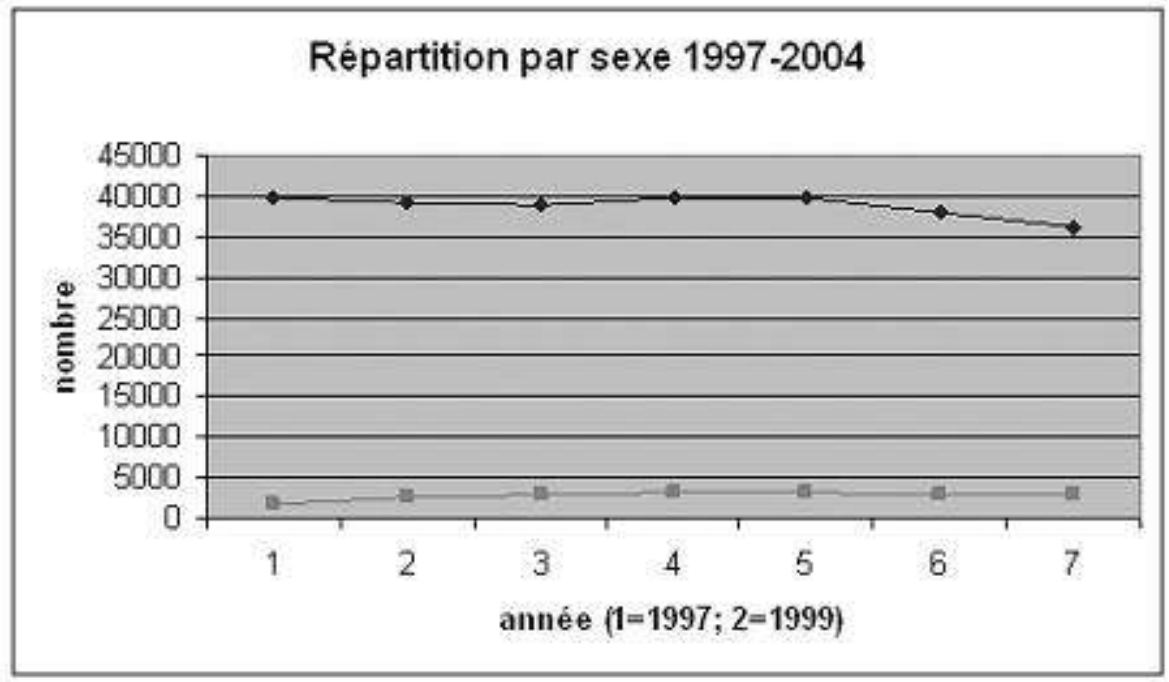

Source : ibid.

Depuis quelques années le cheminot fait son entrée dans les publications officielles et dans la publicité de la SNCB. Le rapport d'activité annuel de la SNCB entre 1997 et 2004 (non paru en 1998) indique bien cette évolution. En 1997, $11 \%$ des photographies incluses dans cette publication montrent le personnel au travail. En 1999 ce pourcentage augmente. Le nombre absolu de photographies double à partir de 2002, mais la proportion d'images du personnel ne suit pas cette augmentation. Un an plus tard, le nombre absolu de photographies du personnel marque une forte augmentation, mais en nombre relatif la représentation est stabilisée à presque $25 \%$ de l'ensemble. Parmi les représentations du personnel, les agents de train sont plus présents que les autres professions. Dans l'ensemble, on voit davantage de voyageurs. L'élément commercial et le service à la clientèle sont donc mis en évidence.

Tableau 3. La présence du personnel dans les rapports annuels publiés par la SNCB, 1997-2004 (nombre de photographies)

\begin{tabular}{|l|l|l|l|l|}
\hline Année & $\begin{array}{l}\text { Nombre total de } \\
\text { photographies illustrant les } \\
\text { rapports }\end{array}$ & $\begin{array}{l}\text { Photographies } \\
\text { représentant } \\
\text { personnel }\end{array}$ & $\begin{array}{l}\text { Part du } \\
\text { total } \\
(\%)\end{array}$ & $\begin{array}{l}\text { Dont personnel du } \\
\text { service des trains } \\
(\%)\end{array}$ \\
\hline 1997 & 28 & 3 & 11 & \\
\hline 1998 & & & 20 & \\
\hline 1999 & 29 & 6 & 17 & \\
\hline 2000 & 35 & 6 & 21 & 0 \\
\hline 2001 & 28 & 6 & 14 & 35 \\
\hline 2002 & 50 & 7 & & \\
\hline
\end{tabular}




\begin{tabular}{|l|l|l|l|l|}
\hline 2003 & 95 & 24 & 25 & 29 \\
\hline 2004 & 90 & 21 & 23 & 38 \\
\hline
\end{tabular}

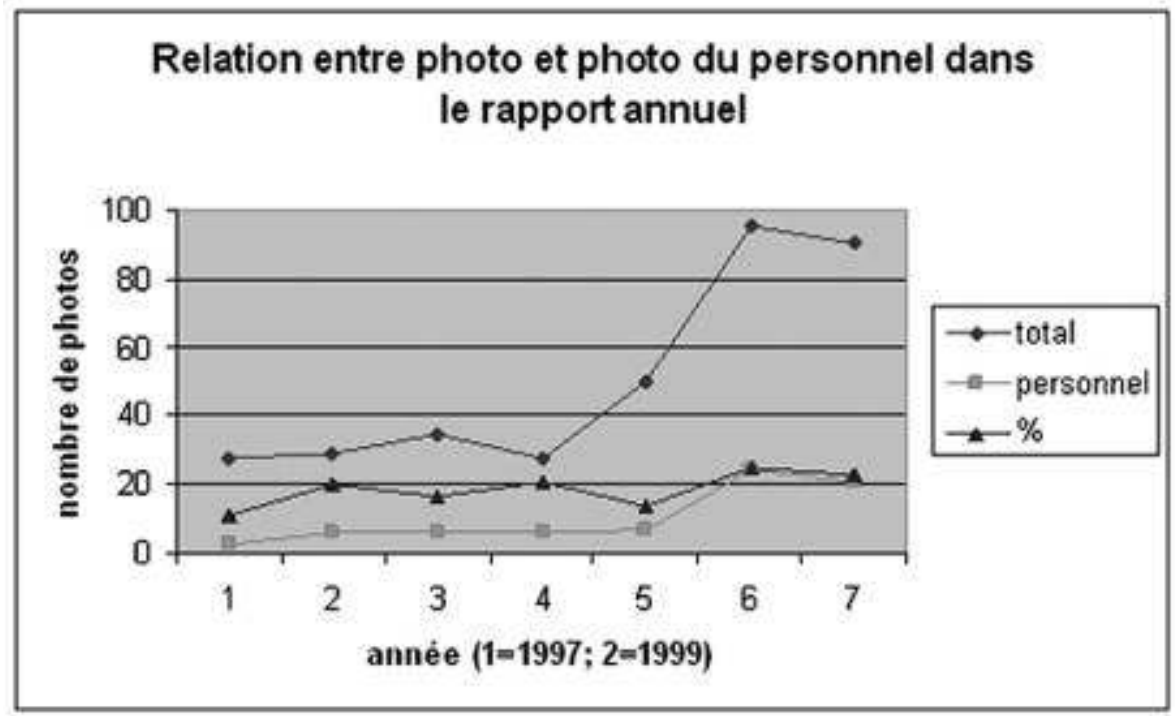

Source : ibid.

Le résultat de cette petite enquête ne permet pas d'établir une périodisation de la représentation, mais il est clair que, dans les quinze dernières années, l'image et la représentation des cheminots ont changé. L'invisibilité des cheminots appartient à l'histoire, mais ce sont surtout les professions spécifiques du service à la clientèle qui sont devenues plus visibles. L'absence de l'image du travail pendant une très longue période n'est pas caractéristique de la Belgique. Michel Ionascu rappelle que le train est l'invention technique la plus marquante du siècle, avec un engin moteur très photogénique; or les cheminots n'en ont pas pour autant été des personnages importants des reportages ou des fictions. Le grand absent, c'est le travail. Il cite Patrick Farbiaz :

Les ouvriers et ouvrières des petites et grandes entreprises, les salariés au SMIC ne font pas des exclus de la société, et en ce sens, ils ont un statut social, participent à la vie économique et sont partie intégrante de nombreuses institutions et appareillages institutionnels et sociaux. Mais l'exclusion médiatique dont ils sont l'objet, qui frappe leur condition trop banale, leurs traditions sociales et leur culture, invalide leurs formes de sociabilité, prive leurs discours de légitimité, les marginalise. Le téléspectateur ouvrier sommé de renier son identité ouvrière, n'a qu'une alternative : s'aligner sur le standard de la classe moyenne mise en scène à la télévision ou rejoindre les sans paroles, les exclus sociaux, clochards et sans domicile fixe ${ }^{7}$.

La présence de certaines professions est une construction de la SNCB qui veut contrôler son image d'entreprise. L'image du cheminot est rare, les mannequins sont très présents. Dans ce sens, il s'agit d'une représentation construite selon des conventions 
du management. Mais il ne s'agit pas de la représentation du travail. Dans la plus récente campagne de recrutement, un homme et une femme sont représentés pendant qu'ils construisent un chemin de fer en miniature. Au lieu de montrer les métiers de la construction, l'image nous fournit un couple en train de jouer avec un modèle réduit.

En quinze ans le spectateur se retrouve donc décidément dans un autre univers: l'image des cheminots, presque inexistants voici vingt ans, a évolué vers une visibilité plus explicite, vers une représentation moins masculine. Le visible et le lisible des chemins de fer, c'est la technologie et cet élément n'a guerre changé, mais l'homme y a sa place, le cheminot autant que le voyageur. Les cheminots sortent tout doucement des coulisses, avec une image qui ne gêne plus ; l'héroïsme n'y est plus, nous vivons une époque de démythification, qui entraîne la création d'autres mythes. Le cheminot n'est plus le mâle (machiste) ou le chef de train trop sérieux. La SNCB prend soin de son image, en utilisant des modèles et des mannequins. Ce ne sont pas des cheminots et le vrai est donc un faux, mais fait d'une manière à être crédible et digne de foi, afin de donner l'impression que le déplacement en train est de nouveau "sexy ». La récente publicité de la SNCB lors de l'ouverture de la ligne nouvelle vers l'aéroport de Zaventem, Brussels Airport, montre la liaison (intime) entre un agent de train et une hôtesse de l'air. Cette image était impossible et impensable voici dix ans. Si la construction de l'image est plus éloignée de la réalité que jamais, au moins cette image existe.

Comment le cheminot évoluera-t-il ? C'est difficile à dire, mais cette évolution aura une répercussion certaine sur sa représentation. La culture d'entreprise de jadis, pour autant qu'elle existe encore, disparaîtra probablement à grande vitesse; l'identité actuelle du cheminot disparaîtra au même rythme. Le plus grand changement viendra de l'évolution interne des systèmes de transports et de leur dynamique propre vis-à-vis de l'utilisateur: les entreprises répondent au désir de la clientèle ; à long terme, les systèmes collectifs vont évoluer vers des services plus individualisés. Les systèmes individuels par contre vont évoluer dans le sens inverse. Cet élément dans tous les cas va altérer l'image et l'identité et les remplacer par d'autres. Si cette hypothèse est exacte, c'est bien le monde extérieur qui, pour une part, construit l'identité des cheminots. Il est donc peu probable que le cheminot et son identité disparaissent.

\section{NOTES}

1. Michel Ionascu, Cheminots et cinéma. La représentation d'un groupe social dans le cinéma et l'audiovisuel français, Paris, L'Harmattan, 2001, p. 9.

2. Ibid.

3. Piet Buunk, Het imaginaire verleden. Beeldende kunst en geschiedschrijving [Un imaginaire du passé. Les arts plastiques dans l'historiographie], Kampen, Kok Agora, 1994, 338 p. Cet ouvrage remarquable omet pourtant le roman de Gustaaf Vermeersch, dont je parle plus bas. Voir aussi W. Hansen, «Introduction », dans Piet Buunk (dir.), Bestijg nooit de trein zonder uw valies met dromen. Gedichten, Verhalen, Columms vanaf 1829, [Ne montez 
jamais dans le train sans vos bagages plein de rêves. Poèmes, contes, feuilletons à partir de 1829], Amsterdam, De Bezige Bij, 1989, p. 5-23.

4. Michel Ionascu, op. cit., p. 9.

5. Hugo De Bot, Architecture des gares en Belgique, 1835-1914, Turnhout, Brepols, 2002, 240 p. et Architecture des gares en Belgique, 1914-2003, ibid., 2003, 207 p. Voir le compte rendu de ces ouvrages sur www.trains-fr.org/ahicf.

6. Paul Van Heesvelde. "A Rolling Life". Gustaaf Vermeersch and the Belgian Railways, paper presented at the 3th $\mathrm{T}^{2} \mathrm{M}$ Conference, York, 2005.

7. Michel Ionascu, op. cit., p. 332-333 ; la citation est de Patrick Farbiaz, Télévision : programmes et exclusions sociales, dans Politis, $\mathrm{n}^{\circ} 3$ (juin 1993).

\section{RÉSUMÉS}

L'analyse des images, photos ou films présentant des cheminots n'a pas encore été faite en Belgique. Cet article présente à titre préliminaire une vision d'ensemble de l'image et l'identité cheminotes belges, premier aperçu d'un champ de recherche resté inexploité. Cependant, des indices convergents nous permettent d'affirmer que l'image belge du cheminot n'est pas très éloignée de l'image française de la même corporation. Nous nous limitons à l'analyse de quelques photographies, qui toutes escamotent les cheminots plus qu'elles ne les dévoilent. Nous voyons ensuite comment l'image de quelques professions cheminotes est récemment entrée dans les publications officielles de la SNCB et évaluons ce qui reste de la mémoire collective, de la création d'identité, de l'esprit d'équipe et de la culture d'entreprise antérieurs dans une société de transports qui s'est scindée, sous la pression de la législation européenne, en trois entreprises distinctes. Dans tous les cas, on peut se demander si la façon de représenter les cheminots transmet une image exacte des hommes et des femmes qui font circuler les trains. L'enseignement final sera de méthode: à défaut d'un corpus riche de grandes séries, il faut utiliser des sources nouvelles pour construire une image dont la cohérence sera finalement plus ou moins assurée.

The critical analysis of Belgian photographs or film images of railwaymen remains to be done. To that end, this article is a preliminary study that presents an overview of the image and identity of the Belgian railwayman, a first step into a relatively unexplored research field. Converging information leads us to confirm that the Belgian image of the railwayman is quite similar to the image of his French counterpart. We have limited our study to the analysis of a few photographs that all tend to camouflage the railwayman more than reveal his identity. We then see how the image of a few of the railwayman's occupations have been adopted in official SNCB publications and evaluate the remnants of their pre-existing collective memory, creation of identity, team spirit and business culture as employees of a transportation firm pressured by European legislation to divide itself into three different companies. One might ask if the way railwaymen are represented conveys an accurate image of the men and women who actually make the trains run. The final lesson concerns methodology: in the absence of a rich and abundant collection of material, we must turn to new sources before constructing a definitive image that is more or less reliable. Résumé traduit du français par Kenneth Berri. 
INDEX

Mots-clés : Belgique, cheminot, cinéma, film, photographie

Keywords : belgium railwayman, cinema, film, photography

\section{AUTEUR}

PAUL J.G.M.J. VAN HEESVELDE

Historien 